\title{
Evaluating the impact of results-based motivating system on noncommunicable diseases risk factors in Iran: Study protocol for a field trial
}

\author{
Mehran Asadi-Aliabadi ${ }^{1}$, Arash Tehrani-Banihashemi ${ }^{1,2}$, Fariba Mirbaha-Hashemi ${ }^{1}$, Leila Janani ${ }^{1,3,4}$, Ebrahim Babaee ${ }^{1}$, \\ Seyed M. Karimi ${ }^{5}$, Marzieh Nojomi ${ }^{1,2}$, Maziar Moradi-Lakeh ${ }^{1,2} *$ (D)
}

Received: 26 Oct 2020

Published: 24 May 2021

\section{Abstract}

Background: Risk factors of noncommunicable diseases (NCD) are increasingly contributing to morbidity and mortality in Iran. Health care providers' competencies and motivation are essential factors for the success and efficiency of primary health care. This field trial aims to evaluate the impact of a results-based motivating system on population level of the NCD risk factors field trial (IRPONT) in Iran.

Methods: Population groups of 24 rural or urban catchment areas from 3 provinces were randomized to 1 of the 4 types of study groups. The groups were defined based on a set of 4 intervention packages. Extra 8 rural or urban catchment areas in a separate city were considered as independent nonintervention (control) group. Population levels of major NCD risk factors in all 32 population groups were measured at the beginning of the trial, at the end of the first year, and will be measured in the second year through standardized population surveys. As the outcome measure, the difference in population levels of the risk factors will be compared among the study groups. Study group IV will be compared with combined control groups (study groups I, II, and III). Also, we will conduct subgroup analysis to determine the effects of interventions 2, 3, and 4.

Ethics: This trial has received ethical approval from National Institute for Medical Research Development in Iran (IR.NIMAD.REC.1396.084) in 2017.

Trial Registration Number: This trial has been registered on the Iranian Registry of Clinical Trials (identifier: IRCT20081205001488N2). Registered on 3 June 2018 and updated on 12 April 2020.

Keywords: Pay for performance, Risk factors, Noncommunicable diseases, Trial, Iran

Conflicts of Interest: None declared

Funding: Research reported in this publication was supported by Elite Researcher Grant Committee under award number 958058 from the National Institutes for Medical Research Development (NIMAD), Tehran, Iran. It has been approved by the national committee on ethics in medical research (code: IR.NIMAD.REC.1396.084) as well as our institutional review board (code: IR.IUMS.REC.1395.1057613).

\section{*This work has been published under CC BY-NC-SA 1.0 license.}

Copyright $\odot$ Iran University of Medical Sciences

Cite this article as: Asadi-Aliabadi M, Tehrani-Banihashemi A, Mirbaha-Hashemi F, Janani L, Babaee E, Karimi SM, Nojomi M, Moradi-Lakeh M. Evaluating the impact of results-based motivating system on noncommunicable diseases risk factors in Iran: Study protocol for a field trial. Med $J$ Islam Repub Iran. 2021 (24 May);35:66. https://doi.org/10.47176/mjiri.35.66

\section{Introduction}

Globalization and rapid urbanization have a significant impact on the environment and lifestyle of all people. Aging and unhealthy lif estyle have increased the burden

Corresponding author: Dr Maziar Moradi-Lakeh, moradilakeh.m@iums.ac.ir

1. Preventive Medicine and Public Health Research Center, Psychosocial Health Research Institute, Iran University of Medical Sciences, Tehran, Iran

2. Department of Community and Family Medicine, School of Medicine, Iran University of Medical Sciences, Tehran, Iran

3. Department of Biostatistics, School of Public Health, Iran University of Medical Sciences, Tehran, Iran

4. Clinical Trial Center, Tehran University of Medical Sciences, Tehran, Iran

5. Department of Health Management \& System Sciences, School of Public Health \& Information Sciences, University of Louisville, Kentucky, United States of noncommunicable diseases (NCD), especially in lowand middle-income countries (1). Also, $57 \%$ of all deaths in 1990 were related to NCD (2), which increased to

$\uparrow$ What is "already known" in this topic:

Non-communicable diseases (NCDs) are the leading cause of morbidity and mortality in the world. This is the protocol of a field trial designed to improve NCD risk factor control through motivating and engagement of non-physician healthcare workers.

$\rightarrow$ What this article adds:

In this study protocol, step-by-step implementation of intervention packages are described. The findings of the trial will be presented later. 
$73.4 \%(72 \cdot 5-74 \cdot 1)$ in 2017 . In other words, 41.1 million (40.5-41.5) people died of NCD in 2017 (3).

Population aging and increased exposure to NCD risk factors have increased the global burden of NCD over the past 3 decades $(4,5)$. Likewise, in Iran, the proportion of the elderly population has increased in recent years (6), which could contribute to the increasing incidence of NCD. According to the World Health Organization, 82\% of all premature mortality in Iran was attributable to NCD in 2016 (7), a warning signal for the urgent need for better planning and implementation of prevention and control programs for NCD. Furthermore, given the limited available resources, there is an urgency for prioritizing more effective programs with lower costs (8). Compared to individual-based interventions, population-based interventions are less costly and are specifically suitable for countries with low resources (9). On the other hand, considering the high prevalence of NCD risk factors in the community, making even minor changes in the population level of the risk factors among the population will be of great benefit to public health.

There are several strategies for implementing prevention programs and controlling the risk factors of NCD. Involving the population to understand the risk and empowering them to participate in planning and operating programs to reduce the NCD risk factors are among the most important strategies (10). Such activities crucially depend on the active role of health care providers to lead population mobilization and do advocacy for collaboration of other sectors. Local health care providers should be motivated enough to take this responsibility. One of the strategies to encourage health care providers is performance-based payment (P4P) or result-based financing, which is an intervention designed to increase the quantity and quality of health care services (11). P4P programs can be applied to both demand and supply sides of health care market (12). Supply-side incentives usually target health care managers, health care providers, or their employees. They incentivize for achievement of predetermined performance indicators, specified in an executive agreement (13). Several studies have shown satisfactory results of supply-side incentives in low- and middle-income countries (14-16). However, these studies have significant limitations in their methodologies. For example, they lack control groups and/or are potentially affected by confounders (14-16).

Nonetheless, there is no certainty about the impact or sustainability of P4P effects over time $(17,18)$. Therefore, potential sustainability risks must be identified. Some common risks are as follows (19):

- Paying less attention to the activities that are not incentivized;

- Change in providers' focus from "hard-to-reach" groups to more accessible groups, mostly providing unnecessary services to people who do not need them only to achieve the targets;

- Change in incentives because of geographic inequality in the availability of resources (eg, health care providers may be better able to reach the targets in less deprived areas, and so they are more likely to receive rewards in these areas);
- Falsification of indicators by healthcare providers.

In this paper, we provide the protocol impact of a results-based motivating system on population level of the NCD risk factors field trial (IRPONT). This trial was started and has been continued as scheduled by the time of submission of this publication; however, the unexpected COVID-19 pandemic situation and its related national or local restrictions have affected the trial in the second year. The findings and process of the trial will be presented in future publications.

\section{Methods}

This study is designed as a field trial. It is performed through formal collaborations with 3 universities of medical sciences in Iran. Universities of medical sciences in Iran are responsible for the health of the population for a defined catchment area. The 3 collaborating universities (Iran University of Medical Sciences, Semnan University of Medical Sciences, and Bushehr University of Medical Sciences) cover a total population of 6.7 million individuals with different levels of socioeconomic status.

Iran has a hybrid health care and medical education system. Its health system is organized in 3 levels of district, province, and district. At the national level, the Ministry of Health and Medical Education (MOHME) is the steward of health system and is responsible for policymaking, planning, directing operations, and overseeing the work of health care organization as well as education of health sciences. Each province has at least 1 local "university of medical sciences." The university chancellor is the highest rank person of the health system in the catchment area of the university. Each university of medical sciences has specific deputies for public health, treatment affairs, and food and drug, in addition to deputies for education and research and technology as the innate roles of each university. Universities of medical siciences are responsible for planning, directing, and supervising health network in the catchment area, which includes supervision on the governmental, public nongovernmental, and private health organizations. The head of the health network in each district, which covers urban and rural areas, is assigned by the university chancellor. The system in general is centralized, but each level has some authorities to initiate specific programs. At each level, there are possibilities for intersectoral collaborations and community participation to improve population health, although those capacities are usually underutilized (20-22).

Patient and Public Involvement: In our field trial interventions, researchers do not have any direct contact with patients or public in the catchment areas. Our interventions affect health care workers, and we expect patients and people who refer to our selected centers to be impacted by our interventions. We do not incorporate or offer any change in the protocols for clinical work. All of our interventions aim toward encouraging primary health care providers to do activities, such as advocacy with community leaders, enabling and engaging people, and doing active follow-ups for people with NCD risk factors, to achieve the predefined goals for population health. We interviewed a few of health care workers and different 
levels of managers for designing the interventions as well as getting a sense of incentives that are encouraging to health care providers. We used their comments to improve our methods. The protocol of this study is available on Iranian Registry of Clinical Trials both in Persian and English languages. Also, a summary of the project and links to trial registry, ethical approval, and list of team members are available in the website of our research center.

Interventions and study groups :In this trial, we have 4 types of interventions in the local health system.

\section{Intervention 1}

Assessment of the main NCD risk factors and setting time-bound targets. Although some data on NCD risk factors are routinely collected in the health network or as periodical surveys in national and province levels, the assessment of NCD risk factors at the local community level is not a part of the routine health network activities. We provided the health care staff with a list of indicators (selected through results chain approach, from output, outcome or impact, and considering the availability and timeliness of routine data) and a manual for measuring the indicators. Researchers, local health authorities, and health staff will participate in setting appropriate time-bound targets for each field. We also made sure that all essential devices for NCD risk factor control in all selected fields are available and functional as part of this intervention. This intervention is performed for all study groups (study groups I, II, III, and IV) (Table 1).

\section{Intervention 2}

Finding evidence on effective and efficient interventions for controlling the risk factors of NCD and sharing the evidence with health care staff and health care authorities through educational workshops and materials. We performed a review to obtain the best available evidence and best practices to control NCD risk factors. This information was shared with the health care authorities and health care staff of the study groups II, III, and IV through several standardized workshops. Intervention 2 is conducted only to provide the evidence; any decision for selection, adoption, and execution of interventions are made by the health care staff and local authorities and population. A free demand-based consultation service (by the researchers) is also available for the health care authorities and staff to help them with the process. We provide them a supportive budget for running the interventions, but they are responsible for all related activities, including community engagement, intersectoral collaboration, and fundraising.

\section{Intervention 3}

Operational planning with the contribution of local health authorities and health care staff to meet the predefined targets of the first intervention. Health care staff and local health care authorities and researchers actively collaborate to plan the activities, and in addition to the technical consultation, monitoring activities are performed by the researchers. This intervention is only done for the study groups III and IV. Unlike the consultation in intervention 2, which is passive and demand-based, the research team is actively involved in this phase to ensure that study groups III and IV have their own operational plans.

\section{Intervention 4}

Implementation of a health workforce result-based motivating system, which will be done only for the study group IV. We were open to sellect the type of the financial motivational incentives with a budget cap. Group discussion with members of the Group IV and local health care authorities led us mainly toward the financial motives. We used a defined available mechanism, such as overtime payment and remuneration, for extra payments. However, we used the research budget to pay for meeting the targets. We clarified for the relevant staff that these incentives are given if they meet the predefined objectives of the first intervention. The nonphysician health care workers (NPHWs) (ie, community health care workers, Behvarzes, and health caregivers who are usually from midwifery, nursing, or public health background) are recipients of this type of incentives. An initial investment tranche (from the budget of our trial) is used to cover the costs of incentives. We use a list of results chain indicators from the routine health indicators (SIB systeml) to give rewards every 3 months, but we will use independently collected data through surveys to assess the efficacy of the interven$\operatorname{tion}(\mathrm{s})$.

Table 1. Study groups: interventions, and number in each district and the entire study

\begin{tabular}{|c|c|c|c|c|c|c|c|c|}
\hline \multirow[t]{3}{*}{ Study groups *** } & \multirow{2}{*}{\multicolumn{4}{|c|}{ Interventions* }} & \multicolumn{4}{|c|}{ Number of fie:lds } \\
\hline & & & & & \multicolumn{2}{|c|}{ Each district } & \multicolumn{2}{|c|}{ Total } \\
\hline & 1 & 2 & 3 & 4 & Urban & Rural & Urban & Rural \\
\hline $\bar{I}$ & Yes & No & No & No & 1 & 1 & 3 & 3 \\
\hline II & Yes & Yes & No & No & 1 & 1 & 3 & 3 \\
\hline III & Yes & Yes & Yes & No & 1 & 1 & 3 & 3 \\
\hline IV & Yes & Yes & Yes & Yes & 1 & 1 & 3 & 3 \\
\hline
\end{tabular}

*Interventions 1: Setting time-bound targets to reduce NCD risk factors in study group's I-IV, Interventions 2: Education of health care workers in study groups II-IV, Interventions 3: Collaborative operational planning to achieve targets in study groups III-IV and Interventions 4: Result-based financial motivation system only in the study group IV. Non-financial supports (gifts and letter of participation) is provided to all groups.

** The "no-intervention control group" is not included in the table; it consists of 4 rural and 4 urban fields in a separate city. Only annual surveys are conducted for this group. 
Criteria for Selecting Medical Universities and Districts

Iran has an integrated health care and education system. Each province in Iran is covered by at least 1 public medical university, and the chancellor of the medical university is the steward of health in the catchment area. Public medical universities are classified in 3 different tiers, which are closely related to the level of development of the catchment area, socioeconomic status of the covered population, and the size and history of universities. We purposefully selected 3 public medical universities, each from 1 of the 3 tiers, with at least 1 district that covers no less than 4 rural fields and 4 urban fields, and their relevant authorities (the vice-chancellor for health) were interested to collaborate in this research. The selected medical universities and districts include Iran University of Medical Sciences as a tier 1 university (Shahriar district), Semnan University of Medical Sciences as a tier 2 university (Damghan district), and Bushehr University of Medical Sciences as a tier 3 university (Dashtestan district).

We were worried about the contamination of study groups by intervention in other fields of the same district. Hence, a fourth district (city of Garmsar in Semnan province) was added to the study. None of the interventions, 1 to 4 , was done in this district, and it was named the nonintervention control group.

Formal bilateral memorandum of understanding documents was signed by the authorities of the collaborating universities to clarify roles and responsibilities of each side and to assure sustainability of the processes.

\section{The Criteria for Entry of Health Care Fields}

The rural field, with at least 2 health care providers (behvarz: Healthcare providers in the village) and, preferably, without a satellite village, and the urban field, with at least 2 health care providers or (moragheb salamat), were considered for this study. Before listing the eligible fields and randomization, we excluded fields with specific characteristics that differentiate them from other fields in each district.

In the next step, from the list of eligible health houses in the rural areas and health bases in the urban areas of each district, 4 rural and 4 urban fields were randomly selected. In each of the first 3 districts (Shahriar, Damghan, and Bushehr), selected fields were randomly allocated to 1 of the study groups I to IV, stratified by rural or urban. For the fourth district (Garmsar), we selected 4 rural and 4 urban fields, but as mentioned earlier, there was no need to allocate them to the study groups.

\section{Surveys}

We performed a baseline population-based survey before starting the interventions. The surveys will be repeated at 12th and 24th months after starting the interventions. We presume that changing some of the behaviors (such as smoking, physical activity, and low intake of fruit and vegetables) takes time, and 1 year of follow-up might not be enough to detect changes. On the other hand, we need to show the sustainability of potential changes. Some of the primary improvements might regress after a while.
Collected data will be analyzed through time series analysis. Study group IV will be compared with combined control groups (study groups I, II, and III). Also, we will do subgroup analysis to determine the effects of interventions 2,3 , and 4 .

Individuals aged 30 to 70 years who have been registered as residents of the catchment areas of the selected urban and rural health fields are eligible for the survey. Providing informed consent is a necessity to include participants in the surveys.

The sample size in each survey (in each of the districts) was estimated to be 320-stratified by the urban and rural study groups, sex (male and female), and 4 age groups (30-39, 40-49, 50-59, 60-70). Sampling is done equally on any sex-age class. Five individuals from each sex in each of the age groups 30 to 39,40 to 49,50 to 59 , and 60 to 70 years should be included in the study.

Subsequently, a list of household codes was obtained from each of the study fields (urban and rural fields in each district), and 32 household codes were randomly selected as the head of the cluster. From the right of the place of that household, the survey continued to reach the desired sample size. Only 1 person from each of the age/sex groups can participate in the study in each household.

\section{Questionnaire and Interview Guide}

In this study, the Persian translation of the World Health Organization Stepwise survey questionnaire (WHO STEPs), previously used in the national STEPs $(23,24)$, was used to collect data. This questionnaire has 3 steps in behavioral, physical, and biochemical measurements. We prepared a manual in the Persian language for the interviewers and trained them in similar standardized workshops in each district for asking the questions, doing the required physical exams, and referring the participants to specific laboratories or health house (in some remote rural areas) for blood and urine sampling. All interviewers were from local residents with relevant education and were trained for the survey. Survey teams of 2 persons (1 female and 1 male) to the households to consider same-sex questioning and physical measurements.

\section{Physical Measurement Instruction}

Physical measurements include standardized measuring of height, weight, waist circumference (WC), hip circumference (HC), and blood pressure. Measuring devices can be used well after assuring the correct operation of the measuring instruments. Measurement tapes were used for WC, HC, and height (attached to the wall for height). Digital weight scale (EmsiG BD46) was used to measure weight, and the M6 comfort Omron digital sphygmomanometer was used for measuring blood pressure. Blood pressure was measured 3 times with 10 minutes difference during the interview, and the average was registered for each participant.

\section{Biochemical Measurement Instructions}

After the last question, the interviewers need to give the participants a referral card and explain the blood and urine 
tests to them. Participants should follow the instructions and go to the designated laboratory, or health houses, which is indicated on the referral card in the date and time specified by the interviewers. The following tests are performed: glycated hemoglobin (A1C), fasting blood sugar, total cholesterol, high-density lipoprotein cholesterol, lowdensity lipoprotein cholesterol, triglycerides, and sodium urine test (random).

In order to separate plasma, the tubes for all blood tests should be centrifuged at $4^{\circ} \mathrm{C}$, with a force of $3000 \mathrm{RPM}$ for 20 minutes immediately after taking the samples. After preparing the samples from all 4 health care networks with the observance of cold chains and transportation principles, biological samples are transferred to the central laboratory in Tehran, Iran.

\section{Primary Outcome}

The primary outcome measures of the study will be the differences in population-level mean and proportion change (from baseline) in uncontrolled hypertension, poorly controlled diabetes, insufficient intake of fruits and vegetables, insufficient physical activity, current tobacco smoking, high salt intake, and body mass index (obesity and overweight). These variables will be measured at the beginning of the intervention, 12 and 24 months after the start of the interventions.

\section{Costs}

In this study, direct and indirect costs from the perspective of the health system are extracted and included in the relevant checklists. Direct costs include the costs that are spent on the different stages of interventions, such as training costs, transportation costs, catering, equipment, and specific costs of interventions. Indirect costs include the opportunity cost of human resources and facilities that are incurred during our study.

\section{Statistical Analysis}

A descriptive analysis of the variables in base survey is performed.Then, weights were calculated and applied based on the city. This process will also run for future surveys. Mean (standard deviation), median, and quantiles will be used to describe the quantitative variables and frequency distribution (\%) for describing qualitative variables. Due to the sampling structure and the random allocation (stratified and clustered), we will use the svyset command for survey data analysis. Methods of difference in difference will be used to compare study groups. We will estimate all measures with their 95\% confidence intervals $(95 \% \mathrm{CI})$; Stata version 14.1 software (StataCorp) will be used in data analyses.

Parallel to the trial, we will calculate the incremental cost-effectiveness ratio (ICER) through comparing each of the II-IV study groups with the study group I:

$$
\mathrm{ICER}=\frac{C_{i}-C_{0}}{E_{i}-E_{0}}
$$

where $C_{i}$ is the cost of the intervention, $C_{0}$ is the cost of the intervention group $I$, and $E_{i}$ and $E_{0}$ are the effects (or change in each of the indicators as measured by the sur- veys) in the intervention and reference groups, respectively.

\section{Trial Status}

After signing the official Memorandum of Understandings with 3 involved universities of medical sciences, we completed the following stages by the end of 2019 :

- Conducting the first survey;

- Randomizing (random assignment) the selected urban/rural areas to 1 of the 4 intervention groups;

- Implementing interventions according to the study protocol for 1 year;

- Conducting the second survey.

The interventions will be continued for the second year, and the third survey will be conducted as the final stage of the trial.

\section{Discussion}

This is the first trial investigating the effects of a resultsbased motivating system for NPHWs on the population level and their interventions on NCD risk factors in Iran. The intervention consists of 4 methods, as stated above, for the prevention and control of NCD risk factors. Iran has an almost unique health system that integrates education of medical sciences and provision of health services; each of the public universities of medical sciences covers a defined catchment area and population, and the university chancellor is the highest health authority in that catchment area (17). In addition to the educational and research responsibilities, each university has vice-chancellors who arrange services for public health, treatment, and food and drug administration. The university is also responsible for accreditation and supervision of health-related private services in its catchment area. Although managers at different levels of health services (eg, province or university level, district level, and urban or rural health center level) theoretically have some authorities for planning and provision of health services; many of the health programs are vertical and are centrally designed at the MOHME (18). It is important for the researchers to understand whether a motivational system for peripheral public health workers could improve population levels of major NCD risk factors in a de facto highly centralized health system. In this trial, there is a differentiation between the NCD control in clients of the health system and the total population in the catchment area. We had to define 4 interventions to differentiate the effect of our motivational system from the effect of proper goal-setting, education, and operational planning enriched with technical support of the researchers.

The areas selected for this study have been stratified based on the socioeconomic background as well as urban and rural locations; this can help us to conclude better about the role of location and regional socioeconomic status.

One of the limitations of this study is the turnover of NPHWs, especially in urban centers; they might go to the regions outside the coverage of this trial, and sometimes they might be relocated to a different intervention group; 
this might happen after the termination of a temporary employment contract or to address human resources in other fields (eg, to cover a health care worker who goes to maternal leave). To address this issue, NPHWs are being tracked closely, and new staff will receive education according to their intervention group. This also might increase the chance of contamination; however, our analysis is based on population groups matched with different study groups (and interventions), not the personnel who are working inside the centers.

In conclusion, by introducing the most appropriate intervention method in this study as a randomized trial, the findings of this study may be beneficial in showing the appropriate path to health policymakers and be effective in preventing and controlling NCD risk factors through lifestyle promotion.

\section{Strengths and Limitations}

- The first interventional study on paying to primary health care providers for performance in Iran, as a country with centralized health system;

- Measuring the effect size based on random sampling of the population of catchment area;

- Using different levels of intervention to separate the importance of content education, supported operational planning, and financial bonus to health care providers;

- Impossibility of blinding primary health care providers;

- Relatively high turnover of primary health care providers, especially in urban health centers and different factors beyond the control of researchers, such as centralized health campaigns, which affect all the country, including our selected fields.

\section{Ethics and Dissemination}

This trial has received ethical approval from Iran University of Medical Sciences (IUMS.REC.1395.1057613. IR) and NIMAD (IR.NIMAD.REC.1396.084) in 2017. All participants of the surveys give written informed consents. Since the interventions of this study need contribution from the personnel of primary health care system, the vice-chancellors of public health in the involved medical universities, as the highest public health authorities in the involved catchment areas, have signed separate memorandum of understandings with the head of Preventive Medicine and Public Health Research Center to officially allow the researchers to implement the interventions. The findings of this study will be published in peer- reviewed journals and conferences; the final report of the study will be submitted to the NIMAD. A policy brief in addition to the final report will be used to inform policymakers and public health authorities at the Ministry of Health and Medical Education of Iran.

\section{Acknowledgement}

The authors would like to thank the collaborators of IRPONT study (Appendix 1), vice-chancellors of Iran University of Medical Sciences (Tehran, Iran), Semnan University of Medical Sciences (Semnan, Iran), and Bushehr University of Medical Sciences (Bushehr, Iran).
Conflict of Interests

The authors declare that they have no competing interests.

\section{References}

1.WHO. Noncommunicable disease and poverty: the need for pro-poor strategies in the Western Pacific Region: a review. Manila: WHO Regional Office for the Western Pacific; 2007.

2.Abubakar I, Tillmann T, Banerjee A. Global, regional, and national age-sex specific all-cause and cause-specific mortality for 240 causes of death, 1990-2013: a systematic analysis for the Global Burden of Disease Study 2013. Lancet (London, England). 2015;385(9963):117-71.

3.Roth GA, Abate D, Abate KH, Abay SM, Abbafati C, Abbasi $\mathrm{N}$, et al. Global, regional, and national age-sex-specific mortality for 282 causes of death in 195 countries and territories, 1980-2017: a systematic analysis for the Global Burden of Disease Study 2017. Lancet. 2018;392(10159):1736-88.

4.Murray CJ, Vos T, Lozano R, Naghavi M, Flaxman AD, Michaud C, et al. Disability-adjusted life years (DALYs) for 291 diseases and injuries in 21 regions, 1990-2010: a systematic analysis for the Global Burden of Disease Study 2010. Lancet (London, England)). 2012;380(9859):2197-223.

5.GBD. Global, regional, and national comparative risk assessment of 84 behavioural, environmental and occupational, and metabolic risks or clusters of risks, 1990-2016: a systematic analysis for the Global Burden of Disease Study 2016. Lancet (London, England). 2017;390(10100):1345-422.

6.Amini R, Ingman S, Sahaf R. Aging in iran: past, present and future2013. 17-34 p.

7.WHO. Noncommunicable diseases country profiles 2018 [Available from: https://www.who.int/nmh/countries/2018/ irn_en.pdf?ua=1.

8.Zulman DM, Vijan S, Omenn GS, Hayward RA. The relative merits of population-based and targeted prevention strategies. Milbank Q. 2008;86(4):557-80.

9.Ha DA, Chisholm D. Cost-effectiveness analysis of interventions to prevent cardiovascular disease in Vietnam. Health Policy Plan. 2011;26(3):210-22.

10. WHO. Action plan for the prevention and control of noncommunicable diseases in the WHO European Region. Proceedings of the Regional Committee for Europe 66th Session. 2016.

11. Megan Ireland, Elisabeth Paul, Dujardina B. Can performance-based financing be used to reform health systems in developing countries? Bull World Health Organ. 2011 Sep 1;89(9):695-8.

12. Borghi J, Mayumana I, Mashasi I, Binyaruka P, Patouillard E, Njau I, et al. Protocol for the evaluation of a pay for performance programme in P'wani region in Tanzania: a controlled before and after study. Implement Sci. 2013;8:80.

13. Bruno Meessen, Agnès Soucat, Sekabaraga C. Performancebased financing: just a donor fad or a catalyst towards comprehensive health-care reform? Bull World Health Organ. 2011 Feb 1;89(2):153-6.

14. Basinga P, Gertler PJ, Binagwaho A, Soucat AL, Sturdy J, Vermeersch CM. Effect on maternal and child health services in Rwanda of payment to primary health-care providers for performance: an impact evaluation. Lancet (London, England). 2011;377(9775):1421-8.

15. Meessen B, Kashala JP, Musango L. Output-based payment to boost staff productivity in public health centres: contracting in Kabutare district, Rwanda. Bull World Health Organ. 2007;85(2):108-15.

16. Schwartz JB, Bhushan I. Improving immunization equity 
through a public-private partnership in Cambodia. Bull World Health Organ. 2004;82(9):661-7.

17. Althabe F, Bergel E, Cafferata ML, Gibbons L, Ciapponi A, Aleman A, et al. Strategies for improving the quality of health care in maternal and child health in low- and middle-income countries: an overview of systematic reviews. Paediatr Perinat Epidemiol. 2008;22 Suppl 1:42-60.

18. Petersen LA, Woodard LD, Urech T, Daw C, Sookanan S. Does pay-for-performance improve the quality of health care? Ann Intern Med. 2006;145(4):265-72.

19. Cometto G. Discussion paper on performance-based financing. 200818 April.

20. Esmailzadeh H, Rajabi F, Rostamigooran N, Majdzadeh R. Iran health system reform plan methodology. Iran J Public Health. 2013;42(Supple1):13.

21. Lankarani KB, Alavian SM, Peymani P. Health in the Islamic Republic of Iran, challenges and progresses. Med J Islam Repub Iran. 2013;27(1):42.

22. Mehrdad R. Health system in Iran. JMAJ. 2009;52(1):69-73.

23. Djalalinia S, Modirian M, Sheidaei A, Yoosefi M, Zokaiee $\mathrm{H}$, Damirchilu B, et al. Protocol design for large-scale crosssectional studies of surveillance of risk factors of noncommunicable diseases in Iran: STEPs 2016. Arch Iran Med. 2017;20(9):-.

24. Mohebi F, Mohajer B, Yoosefi M, Sheidaei A, Zokaei H, Damerchilu B, et al. Physical activity profile of the Iranian population: STEPS survey, 2016. BMC Public Health. 2019;19(1):1266. 
Appendix 1

IRPONT collaborators:

Ministry of Health and Medical Education of Iran collaborator:

Dr. Mohammad Reza Rahbar

Iran University of Medical Sciences collaborators, Tehran, Iran:

Dr. Jalil Koohpadezadeh, Dr. Babak Eshrati, Dr. Omid Pournik, Dr. Mohammad Hadi Naseh, Dr. Betul Taefi, Dr. Nasir Dehghan, Dr. Neda Soleimanvandi, Ms. Fatemeh Alborzi, Ms. Ruqieh Alizadeh, Mr. Jaafar Alyari, Ms. Leila Bektosan, Ms. Parastoo Bolourian, Ms. Sahar Hassandoost, Ms. Maryam Hassanzadeh, Mr. Mehdi Mansouri, Ms. Rana Mashakhi, Ms. Parvaneh Mirzaei, Ms. Arezo Mohammad-Salahi, Mr. Abolhassan Mohammadian, Mr. Gholamreza Nemati, Mr. Hassan Nicksima, Ms. Zahra Qahri-Mobser, Ms. Raedeh Rezaee, Ms. Susan Salim, Ms. Maryam Shahverdi, Ms. Sara Sufi, Ms. Khadijeh Tandideh

\section{Bushehr University of Medical Sciences collaborators, Bushehr, Iran:}

Dr. Abdul Mohammad Khajehean, Dr. Mohammad Mehdi Khajehean, Dr. Razieh Hajioni, Ms. Kobra Aghaee, Ms. Elham Ahmadi-Khorram, Mr. Fatah Allah Hajiani, Ms. Akram Ansari-Far, Ms. Esmat Dehghani, Mr. Mohammad-Sadegh Eskandari, Ms. Zahra fakhar, Mr. Ali Gharibi, Ms. Fatemeh Hayat, Mr. Amin Jahanbakhsh, Mr. Rahim Khademi, Ms. Maria Khishdoost-Borazjani, Ms. Jamileh Moarefi, Ms. Kobra Mohammadi, Mr. Hafiz Omidi, Ms. Azadeh Salemi, Mr. Jahanshir Shahneh, Ms. Homeyra Souleimani, Ms. Mina Tanha, Ms. Marzieh Zaker-Hosseini, Ms. Masoumeh Zamani

\section{Semnan University of Medical Sciences collaborators, Semnan, Iran:}

Dr. Jafar Jandaghi, Dr. Mohammad-Naser Rahbar, Ms. Aghababayian Robabeh, Ms. Zahra Aliannejadi, Mr. Mehdi Alikhanian, Mr. Mostafa AmirFakhrian, Ms. Atefeh Ayubi-Far, Ms. Zohreh Badami, Ms. Zahra Barati, Ms. Elham Behnam, Ms. Faezeh Binaean, Ms. Parisa Chitsaz, Ms. Sedigheh Darbndi, Ms. Sareh Faraji-Khorshidi, Ms. Neda Ghandali, Ms. Homeyra Ghezlou, Mr. Hassan Ghorbani, Ms. Haniyeh Hassani, Mr. Hassan Jahanshiri, Ms. Zahra kheirkhahan, Mr. Ghorban Mohammad Khojami, Ms. Sara Khorrami, Ms. Shahrbanoo Khorsi-Damghani, Mr. Mohammad-Reza Khoshkam, Mr. Mehdi Majeri, Ms. Arezou Mansourian, Ms. Somayeh Mohammadi, Ms. Razieh Mokhberian, Mr. Ali Niknejad, Ms. Mina RabieiFar, Ms. Saeedeh Rezai, Ms. Maryam Roshanaee, Mr. Mahmoud Sabzali, Mr. Ali Sadeghi-Moghaddam, Ms. Nahiid Samiie-Rad, Ms. Sabereh Shabiri, Mr. Ahmad Shah-Hosseini, Mr. Mohammad Souri, Mr. Mostafa Talebi, Ms. Masoumeh Touli 